\title{
Some Properties of Green's Matrix of Nonlinear Boundary Value Problem of First Order Differential
}

\author{
Badrulfalah $^{1 *}$, Dwi Susanti ${ }^{2}$, Khafsah J. ${ }^{3}$, Robin Kosasih ${ }^{4}$ \\ Mathematics Department, Faculty of Mathematics and Natural Sciences, \\ Universitas Padjadjaran Bandung, Indonesia \\ *E-mail : badrulfalah@unpad.ac.id
}

\begin{abstract}
This paper discusses Green's matrix of nonlinear boundary value problem of first-order differential system with rectangular coeffisients, especially about its properties. In this case, the differential equation of the form $A(t) x+B(t) x=f(t, x)$ with boundary conditions of the form $B_{1} x\left(t_{0}\right)=$ $a$ and $B_{2} x(T)=b$ which $A(t)$ is a real $m \times n$ matrix with $m>n$ whose entries are continuous on $J=\left[t_{0}, T\right]$ and $f \in C\left[J \times \mathbb{R}^{n}, \mathbb{R}^{n}\right] . B_{1}, B_{2}$ are nonsingular matrices such that $a$ and $b$ are constant vectors. To get the Green's matrix and the assosiated generalized Green's matrix, we change the boundary condition problem into an equivalent differential equation by using the properties of the Moore-Penrose generalized inverse, then its solution is found by using method of variation of parameters. The last we prove that the defined matrices satisfy the properties of green's function. The result is the corresponding the Green's matrix and the assosiated generalized Green's matrix have the property of Green's functions with the jump-discontinuity.
\end{abstract}

Keywords : Green's Matrix, Nonlinear, Boundary, Diffential

\section{Introduction}

Green's matrix is a function. It is used to represent certain terms in the solution of differential system. In the special case, for one differential equation, it is called Green's function. Talking about Green's function, beside it is a solution of the homogeneous differential equation also there are some the related properties. Among others are the continuity, the derivative and the uniqueness of it. As a solution of the homogeneous differential equation, Green's function has discontinuity at certain points. The jumdiscontinuity of Green's function can have different magnitude. The difference follow the form of differential equations or regarded variable[2,5,6,7]. In the other side in [9] , it has been proved uniqueness the solution of nonlinear boundary value problem of firstorder differential system with rectangular coefficients by using method of iterative monotone.

Inspired by $[2,5,6,7,9]$, in this research will be discussed the property of the Green's function that hold on the Green's matrix of nonlinear boundary value problem of first-order differential system with rectangular coefficients. The objective of this research is to prove that the Green's matrix and the assosiated generalized Green's matrix of the relevant boundary value problem satisfy the property of Green's function. 


\section{Research Method}

To prove the properties of Green's function that hold to the Green's matrix and generalized Green's matrix done in four steps. Firstly is to change a nonlinear boundary value problem of first-order differential system with rectangular coefficients into a differential equation that equivalent by using the property of inverse of Moore-Penrose matrix. Then to find the solution by using method of variation of parameters. After that defining Green's matrix and Green's matrix generalized associate. The last proving the properties of Green's function possessed by them.

\section{Result and Discussion}

\section{Prelimineries}

We consider a nonlinear boundary problem of first-order differential system with rectangular coefficients of the form

$A(t) x^{\prime}+B(t) x=f(t, x)$

with boundary condition of the form

$\begin{array}{lll}B_{1} x\left(t_{0}\right)=a & \text { dan } & B_{2} x(T)=b\end{array}$ (1.2)

where $A(t)$ is a real $m \times n$ matrix with $m>n$ that entries are continuous on $J=\left[t_{0}, T\right]$ and $f \in C\left[J \times \mathbb{R}^{n}, \mathbb{R}^{n}\right] . B_{1}, B_{2}$ are nonsingular matrices, $a$ and $b$ are constant vectors.

We will give some definitions and theories of Moore-Penrose matrix inverse .

Definition 1.1: Let $A(t) \in \mathbb{R}^{m \times n} . A^{+}(t)$ is a real $n \times m$ matrix called Moore-Penrose generalized inverse of $A$ if the following conditions satisfied:

(i) $A A^{+} A=A$

(ii) $A^{+} A A^{+}=A^{+}$

(iii) $\left(A A^{+}\right)^{T}=A A^{+}$

(iv) $\left(A^{+} A\right)^{T}=A^{+} A$.

which $A^{T}$ is transpose of $A$.[9]

Definition 1.2: Let $B(t) \in \mathbb{R}^{m \times n} . B(t)$ is called in column space of $A(t) \in \mathbb{R}^{m \times n}$ if there exist some matrix $M(t) \in \mathbb{R}^{n \times n}$ a such that $B(t)=A(t) M(t)$ for every $t$.[9]

Consider an equation $A x=b$,

which is $A \in \mathbb{R}^{m \times n}, m \geq n$.

Lemma 1.3: (a) The following statements are equivalent:

(i) Equation (1.3) is consistent

(ii) $A A^{+} b=b$

(iii) $\operatorname{rank} A=\operatorname{rank}[A: b]$, in equal, $b$ is a linear combination of column of $A$.

(b) The solution, if it exist, is unique if and only if $A$ has $\operatorname{rank}$ full that is $\operatorname{rank} A=n$.

The solution is given by $x=A^{+} b+\left(I-A^{+} A\right) z$, where $z \in \mathbb{R}^{n}$ arbitrary..

The least square solution of (1.3) is $x=A^{+} b$ with $A^{+}=\left(A^{T} A\right)^{-1} A^{T}$ where $m>n$.[9]

42 IEKSAKTA: Berkala IImiah Bidang MIPA 
The following Lemma 1.4 give a boundary value problem that equivalent to (1.1), (1.2).

Lemma 1.4: Let $A(t)$ and $B(t)$ are matrices in (1.1). If $A(t)$ and $A^{-1}(t)$ are nonnegative then

$A(t) x+B(t) x=f(t, x)$ if and only if $x^{\prime}+M(t) x=h(t, x)$ which is $h(t, x)=A^{+}(t) f(t, x)\left[I(t)-A(t) A^{+}(t)\right] z, z \in \mathbb{R}^{n}$

Consider the boundary problem that equivalent to (1.2) that is $x^{\prime}+M(t) x=h(t, x)$ which is $h(t, x)=A^{+}(t) f(t, x)\left[I(t)-A(t) A^{+}(t)\right] z, z \in \mathbb{R}^{n}$

with boundary conditions $B_{1} x\left(t_{0}\right)=a$ dan $B_{2} x(T)=b$

Observe the homogeneous differential equation related to equation (1.4) that is:

$x^{\prime}+M(t) x=0$

Lemma 1.5: (a) Let $X(t)$ is a fundamental matrix for equation (1.6). If $x_{p}(t)$ is the particular solution of (1.4) then $x(t)=x_{p}(t)+X(t) c$

is the general solution of equation (1.4) for every $c \in V_{n}\left[t_{0}, T\right]$. where $c \in \mathbb{R}^{n}$ arbitrary. Any solution of (1.4) has form(1.7). Moreover, the particular solution of (1.4) is given by

$x_{p}(t)=X(t) \int_{t_{0}}^{t} X^{=1}(s) h(t, x) d s$

(b) Let $D$ be a characteristic matrix for the homogeneous equation problem

$x^{\prime}+M(t) x=0$,

and

$B_{1} x\left(t_{0}\right)+B_{2} x(T)=0$

where is $D=B_{1} X\left(t_{0}\right)+B_{2} X(T)$

If $\operatorname{rank} D=r$. Then its index of compatibility (1.9), (1.10) is $n-r$.

Definition 1.6: A boundary problem is incompatible if its index of compatibility is null ( that is the dimension of its solution space is null dimension).[5]

Lemma 1.7 : Let $X(t)$ is the solution of fundamental matrix for (1.9). If it satisfy:

(i) The homogeneous equation (1.9) is incompatible

(ii) Characteristic matrix $D$ for (1.10) is nonsingular

(iii) $\operatorname{rank} B_{1}=n=\operatorname{rank} B_{2}$.

Then the solution of equation (1.4) with boundary condition (1.5) has the particular solution

$x(t)=\int_{t_{0}}^{T} H(t, s) f(s, x) d s+\int_{t_{0}}^{T} G(t, s)\left[I-A^{+}(s) A(s)\right] z d s+X(t) D^{-1}(a+b)$

where $G(t, s)$ and $H(t, s)$ are

$G(t, s)=\left\{\begin{array}{l}X(t) D^{-1} B_{1} X\left(t_{0}\right) X^{-1}(s), s<t \\ -X(t) D^{-1} B_{2} X(T) X^{-1}(s), s>t\end{array}\right.$

and

$H(t, s)=\left\{\begin{array}{l}X(t) D^{-1} B_{1} X\left(t_{0}\right) X^{-1}(s) A^{+}(s), s<t \\ -X(t) D^{-1} B_{2} X(T) X^{-1}(s) A^{+}(s), s>t\end{array}\right.$

43 IEKSAKTA: Berkala IImiah Bidang MIPA 


\section{Main Result}

In this part will be proved the property of Green'n function that satisfied by Green's matrix. Before it we define the Green's matrix and the assosiated generalized Green's matrix as follow.

Definition 2.1: Define a Green's matrix $G(t, s)$ and an assosiated generalized Green's matrix $H(t, s)$ for boundary problem (1.4) and (1.5) as follow:

and

$$
G(t, s)=\left\{\begin{array}{l}
X(t) D^{-1} B_{1} X\left(t_{0}\right) X^{-1}(s), s<t \\
-X(t) D^{-1} B_{2} X(T) X^{-1}(s), s>t
\end{array}\right.
$$

$$
H(t, s)=\left\{\begin{array}{l}
X(t) D^{-1} B_{1} X\left(t_{0}\right) X^{-1}(s) A^{+}(s), s<t \\
-X(t) D^{-1} B_{2} X(T) X^{-1}(s) A^{+}(s), s>t
\end{array}\right.
$$

The fundamental characteristic of the Green's matrix and the assosiated generalized Green's matrix are revealed in the following theorems.

Theorem 2.2: when $G(t, s)$ and $H(t, s)$ are regarded as function of $t$ with $s$ fixed then $G(t, s)$ and $H(t, s)$ have continuousely first derivatives in $\left[t_{0}, s\right)$ and $(s, T]$. At the points $t=s, G(t, s)$ and $H(t, s)$ have upward jump-discontinuity of unit magnitude; those are $G\left(s^{+}, s\right)-G\left(s^{-}, s\right)=I_{n}$ and $H\left(s^{+}, s\right)-H\left(s^{-}, s\right)=I_{n}$.

\section{Proof:}

Firstly shown that $G(t, s)$ is continue and has a continuousely first derivative in $\left[t_{0}, s\right)$ and $(s, T]$ except at $t=s$.

Write $G(t, s)$ defined by (2.1) as follow:

$$
G(t, s)=\left\{\begin{array}{l}
X(t) K_{+}(s), t_{0} \leq s \leq t \leq T \\
X(t) K_{-}(s), t_{0} \leq t \leq s \leq T
\end{array}\right.
$$

where $K_{+}(s)=D^{-1} B_{1} X\left(t_{0}\right) X^{-1}(s)$ and $K_{-}(s)=-D^{-1} B_{2} X(T) X^{-1}(s)$ are matrices independent of variable $t$. Therefore the components of $G(t, s)$ have continuousely first derivative with respect to $t$ in interval $\left[t_{0}, s\right)$ and $(s, T]$.

The last shown that $G$ has an upward jump-discontinuity of unit magnitude.

Observe that

$$
\begin{aligned}
& \lim _{t \rightarrow s^{+}} G(t, s)=\lim _{t \rightarrow s^{+}} X(t) K_{+}(s)=X(s) K_{+}(s) \text { and } \\
& \lim _{t \rightarrow s^{-}} G(t, s)=\lim _{t \rightarrow s^{-}} X(t) K_{-}(s)=X(s) K_{-}(s) \text {. } \\
& \text { Write } G\left(s^{+}, s\right)=\lim _{t \rightarrow s^{+}} G(t, s) \text { and } G\left(s^{-}, s\right)=\lim _{t \rightarrow s^{-}} G(t, s) \text { then we obtain } \\
& G\left(s^{+}, s\right)-G\left(s^{-}, s\right)=X(s) K_{+}(s)-X(s) K_{-}(s) \\
& =X(s)\left[K_{+}(s)-K_{-}(s)\right] \\
& =X(s)\left[D^{-1} B_{1} X\left(t_{0}\right) X^{-1}(s)+D^{-1} B_{2} X(T) X^{-1}(s)\right] \\
& =X(s) D^{-1}\left[B_{1} X\left(t_{0}\right)+B_{2} X(T)\right] X^{-1}(s) \\
& =X(s) D^{-1} D X^{-1}(s) \\
& =X(s) I_{n} X^{-1}(s) \\
& =I_{n} \text {. }
\end{aligned}
$$

The continuity and existence of its first derivative as well as an upward jumpdiscontinuity of $H(t, s)$ at the points $t=s$ can be proved similarly. We leave it. 
The next Theorem 2.3 we show that both of the Green's matrix and the assosiated generalized Green's matrix are the solutions of the homogenous boundary problem.

Theorem 2.3: If $G(t, s)$ and $H(t, s)$ are regarded as a function of $t$ then both are the solution formal of the homogenous boundary problem (1.9) and (1.10) excep at $t \neq s$.

Proof:

Describing $G(t, s)$ defined by formulation (2.1) is the matrix solution of the homogeneous equation(1.9) except at points $t=s$, that is

$$
\begin{aligned}
G_{t}(t, s)+M(t) G(t, s) & =X^{\prime}(t) K_{+}+M(t) X(t) K_{+}, s<t \\
& =\left[X^{\prime}(t)+M(t) X(t)\right] K_{+} \\
& =O_{n} K_{+} \\
& =O_{n} .
\end{aligned}
$$

and

$$
\begin{aligned}
G_{t}(t, s)+M(t) G(t, s) & =X^{\prime}(t) K_{-}+M(t) X(t) K_{-}, t<s \\
& =\left[X^{\prime}(t)+M(t) X(t)\right] K_{-} \\
& =O_{n} K_{-} \\
& =O_{n} .
\end{aligned}
$$

Moreover,

$$
\begin{aligned}
B_{2} G(T, s) & =B_{1} X\left(t_{0}\right) K_{-}+B_{2} X(T) K_{+} \\
& =\left[D-B_{2} X(T)\right] K_{-}+B_{2} X(T) K_{+} \\
& =D K_{-}-B_{2} X(T) K_{-}+B_{2} X(T) K_{+} \\
& =-D D^{-1} B_{2} X(T) X^{-1}(s)+B_{2} X(T)\left(K_{+}-K_{-}\right) \\
& =-I_{n} B_{2} X(T) X^{-1}(s)+B_{2} X(T)\left[D^{-1} B_{1} X\left(t_{0}\right) X^{-1}(s)+\right. \\
D^{-1} B_{2} X(T) & \left.X^{-1}(s)\right] \quad \\
& =-B_{2} X(T) X^{-1}(s)+B_{2} X(T) D^{-1}\left[B_{1} X\left(t_{0}\right)+B_{2} X(T)\right] X^{-1}(s) \\
& =-B_{2} X(T) X^{-1}(s)+B_{2} X(T) D^{-1} D X^{-1}(s) \\
& =-B_{2} X(T) X^{-1}(s)+B_{2} X(T) I_{n} X^{-1}(s) \\
& =-B_{2} X(T) X^{-1}(s)+B_{2} X(T) X^{-1}(s) \\
& =O_{n} .
\end{aligned}
$$

For $H(t, s)$ can be proved similarly. We leave it.

The last we prove uniqueness of the Green's matrix and the assosiated generalized Green's matrix.

Theorem 2.4: $G(t, s)$ and $H(t, s)$ are unique having properties of Theorem 2.2 and Theorem 2.3.

\section{Proof:}

For $s$ arbitrary, let $K(t, s)$ is others Green's matrix that has properties of Theorem 2.2 and Theorem 2.3.

Write $Y(t, s)=K(t, s)-G(t, s)$. It will be shown that $Y(t, s)=O_{n}$.

It is obvious that $Y(t, s)$ has the first derivative continue with respect to $t$ in interval $\left[t_{0}, s\right)$ and $(s, T]$. Since discontinuity of $K$ and $G$ cancel each other then

$$
\begin{aligned}
Y\left(s^{+}, s\right)-Y\left(s^{-}, s\right) & =\left[K\left(s^{+}, s\right)-G\left(s^{+}, s\right)\right]-\left[K\left(s^{-}, s\right)-G\left(s^{-}, s\right)\right] \\
& =K\left(s^{+}, s\right)-K\left(s^{-}, s\right)-\left[G\left(s^{+}, s\right)-G\left(s^{-}, s\right)\right]
\end{aligned}
$$




$$
\begin{aligned}
& =I_{n}-I_{n} \\
& =0_{n} .
\end{aligned}
$$

Consequently $Y$ has removable discontinuity at the points $t=s$, so with defining $Y$ correctly at the points $t=s$ then it will be obtained $Y$ that continue in $\left[t_{0}, T\right]$. Since $Y$ is a linear combination of $K$ and $G$ then $Y$ is also the solution matrix of the homogeneous equation (1.6) and therefore $B_{1} Y\left(t_{0}, s\right)+B_{2} Y(T, s)=B_{1}\left[K\left(t_{0}, s\right)-G\left(t_{0}, s\right)\right]+$ $B_{2}[K(T, s)-G(T, s)]$

$$
\begin{aligned}
& =B_{1} K\left(t_{0}, s\right)+B_{2} K(T, s)-\left[B_{1} G\left(t_{0}, s\right)+B_{2} G(T, s)\right] \\
& =0_{n}-0_{n} \\
& =0_{n}
\end{aligned}
$$

then for every $s, Y$ is a solution of the homogeneous boundary problem (1.6).

Say $Y(t, s)=X(t) c$, for $c$ is $n-$ constant vector arbitrary.

In view of $B_{1} Y\left(t_{0}, s\right)+B_{2} Y(T, s)=0_{n}$ we get $B_{1} X\left(t_{0}\right) c+B_{2} X(T) c=0_{n}$

$$
\begin{aligned}
D c & =0_{n} \\
D^{-1} D c & =0_{n} \\
I_{n} c & =0_{n} \\
c & =0_{n}
\end{aligned}
$$$$
\left[B_{1} X\left(t_{0}\right)+B_{2} X(T)\right] c=0_{n}
$$

So $Y(t, s)=X(t) c=X(t) 0_{n}=0_{n}$.

Hence $K(t, s)=G(t, s)$.

It means that $G$ is unique.

The uniqueness of $H$ can be proved similarly. We leave it.

\section{Conclusion}

In this paper we have the conclusion that the Green's matrix and the assosiated generalized Green's matrix satisfy the property of Green's function and they have an upward jump-discontinuity of unit matrix magnitude at the points $t=s$.

\section{References}

[1] A. S. Delf. 1982. Advanced Matrix Theory for Scientists and Engineers. Abacus Press. London.

[2] Badrulfalah. 1992. Masalah Syarat Batas Dua Titik. Thesis Program S2 Matematika ITB. Tidak Dipublikasikan. Bandung. ITB.

[3] Boyce, E., William and Diprima, C., Richard.1992. Elementary Differential Equations and Boundary Value Problem ( $5^{\text {nd }}$ Edition). Canada: John Willey \& Sons. Inc.

[4] Braun, Martin. 1982. Differential Equqtions and their Applications (3 ${ }^{\text {nd }}$ Edition). New York. Heidelberg Berlin: Springer-Verlag

[5] Cole, R. H. 1968. Theory of Ordinary Differential Equations. Appleton-CenturyCroft, New York.

[6] Deo, S. G. , V. Raghavendra. 1987. Ordinary Differential Equations And Stability Theory. Tata McGraw-Hill Publ. Co. Ltd., New Delhi.

[7] Murti, K. N., P. V. S. Lakshmi. 1990. On Two- Point Boundary Value Problems. Journal of Mathematical analysis And Applications 153, 217-225. 
[8] P. Lancaster and M. Tismenetsky. 1985. The Theory of Matrices. Academic Press. New York

[9] Uvah J. A. \& Vatsala A. S. 1990. Monotone Iterative Technique for Nonlinear Boundary-Value Problem of First Order Differential System with Rectangulatr Coeffisients. Journal of Mathematical analysis and Applications, 482-49. 